\title{
Object Detection in Camouflaged Environment with Texture Statistical Features
}

\author{
Chennamsetty Pulla Rao, A. Guruva Reddy, C.B. Rama Rao
}

\begin{abstract}
In camouflage image foreground will be hidden in the background image. Camouflage images can be natural and artificial. Detection of such hidden objects becomes difficult for a machine vision system and takes much time to detect and recognize whereas it is not difficult for Human Perception. Detection process involves two phases; feature search that helps in grasping the characteristic entities of an image like colour, shape, texture, pattern etc., and conjunction search which is useful for recognition of clues from multiple features. The background of the camouflage image may be uniform or non-uniform characteristic entities. Different operations can be performed on characteristic entities to make the background disappear in order to detect the foreground image. In this paper, survey on DE camouflaging methods and framework is proposed to detect objects in camouflage environments. Under this framework, textural smoothing followed by statistical characteristics is used to detect the camouflaged objects that will show a better performance based on statistical features. De-camouflaging can be used in war field, where soldiers hide themselves from the enemies with the texture similar to that of their background and decamouflaging is used to reveal the camouflages in the background
\end{abstract}

Keywords-Texture Smoothing, DWT, GLCM, Statistical Characteristics

\section{INTRODUCTION}

Object detection is very simple and less time consuming for human vision applications where as it is tedious and time consuming in the case of machine vision applications under camouflaged environment due to stochastic nature of scene. Detection in such environment is a challenging problem and that will have potential applications. Basically camouflage environment can be divided into two ways, one is artificial and another one is natural. In the case of artificial, we can assess the camouflage whereas in natural, it is highly impossible to assess due to randomness in the scene. Assessment of camouflage can be done in natural camouflage environment with the support of statistical models. Object detection in camouflaged one is often used in different applications by considering a scene or image sequence. Image sequence considered may be a camouflaged one, consisting of foreground and background images [20]. The background image is comprised of static pixels associated with stationary and rest of pixels belonging to non-stationary objects. Each pixel provides information for characterizing either background or foreground. Many changes may be encountered in the background as the time

Revised Manuscript Received on July 10, 2019.

Chennamsetty Pulla Rao, Research scholar, Department of ECE, JNTU, Kakinada, India. (E-Mail: chennamsettypullarao@gmail.com)

Dr.A. Guruva Reddy, Professorl, Department of ECE, India (E-Mail:aguruvareddy@ mictech.ac.in)

Dr.C.B. Rama Rao, Professor, Department of ECE, NIT Warangal, India (E-Mail: cbrr@nitw.ac.in) advances. The background is represented by features at each pixel extracted from the image sequence called image features. Such features are classified as spectral, spatial and temporal features [20]. Spatial and spectral features characterize the presence of static background pixels and recent studies revealed that the temporal features explain about dynamic background pixels. Many methods have been developed for background modeling but fail to represent the complex background consist of both stationary and non-stationary objects [20]. Some of natural camouflage objects detection areas like Detection in reconnaissance system is an important aspect in military war environment and Detection of insects when they try to get camouflage while moving in different natural environment [2] etc.

In 1995 first method suggested was motion camouflage i.e. animals hide from hunter to save themselves [1]. Some animals use counter-shading to hide i.e. in image flat intensity function rather convex [2]. If it is possible to develop a mathematical model for the counter- shading then breaking of camouflage is simple but the mathematical convexity operator can't break the camouflage completely [2]. Meirav Galun suggested texture based segmentation [3] methods and their statistical characteristics in object detection. Civilian applications like manufacturing industry with automation, surgery in medical field etc., detection in camouflaged images system constitutes an important part. The Identification of mingled foreground and camouflaged regions are at different conditions like natural and artificial environments are extensively considered to mitigate the problems in computer vision applications. Many researches are going on these approaches like using came up with different sort of solutions based on characteristic entity based, colour entropy, region based pattern based, classifier based, divergence and background modeling etc. to model a full pledged adaptive model based system for detecting object under camouflage environment due to random characteristic entities (like colour, structure, texture and shape e.t.c.)[20]. These things motivated us to overcome some of the problems. The objective is towards the development of a method completely based on smoothing entities there after statistical characterization of decomposed camouflaged image.

In general the environments like farm fields the crop is effected by insects which are mingled or hide in by similar colors with crop textures. So detecting insects by farmers is difficult in order to minimize the effect in crop production. Also in military environments like enemy soldiers hide with different textures as per the background conditions to do their responsibilities under different war environments (like: green fields, forest, desert and sea etc) to hide from

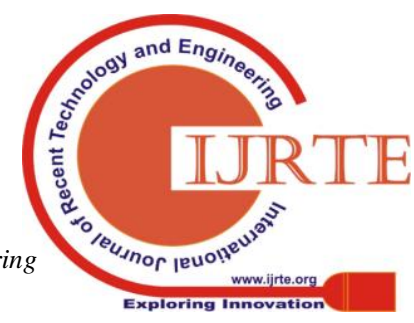




\section{Object Detection in Camouflaged Environment with Texture Statistical Features}

enemies. So the problem can be overcome by the following constructive approach consist of steps like:1) Reading the images form source data base (camopedia) 2) smoothing the characteristic entities like colour, texture e.t.c. 3) Subdivide the equalized image in to different levels in order to evaluate the local statistical features. 4) Evaluate the texture feature from the decomposed image to identify the seed point by taking maximum sum of feature set. 5) Detecting with seed point based region growing approach in image

\begin{tabular}{|c|c|c|}
\hline $\begin{array}{c}\text { Source } \\
\text { data } \\
\text { base }\end{array}$ & $\begin{array}{c}\text { Smoothing } \\
\text { characteristic } \\
\text { Entities }\end{array}$ & $\begin{array}{c}\text { Image } \\
\text { Decomposition }\end{array}$ \\
\hline & & $\begin{array}{c}\text { Statistical Feature } \\
\text { Evolution }\end{array}$ \\
\hline & & $\downarrow$ \\
\hline & & $\begin{array}{c}\text { Camouflaged } \\
\text { Obiect Detection }\end{array}$ \\
\hline
\end{tabular}

\section{Figure 1 Generalized block diagram to detect object}

The main approach of this paper is to model a framework to detect object in camouflaged images and also able to apply on different kinds of images. We constructed a frame work in order to detect camouflage object. The framework consists of a combination of smoothing characteristic entities in camouflage image and statistical characteristics evaluation in decomposed images of different kinds like dimensions, intensity information e.t.c. The recognition can be done in many ways the generalized model is shown in figure 1. In order to smooth characteristic entities, Relative Total Variation is applied [4] and Image decomposition is chosen to decompose in to constituent parts to apply the statistics as it is need of local information context. [3, 8, and 12].

\section{SCOPE OF APPROACH}

There are various methods using different algorithms that are used for background modeling for object detection like Kalman method, Single Gaussian, Mixture of Gaussian, colour and gradient methods, Local Structure, Optical Flow and Non-Parametric. All these methods use either of spatial, spectral and temporal features for background representation and lack in accuracy during object detection and degree of colour entropy is high [20]. None of the developed methods use all the three types of features to represent the background i.e complex backgrounds with both stationary and non-stationary objects are not yet been created using the previously developed methods which needs updating of background due to sudden and once-off changes[20]. Also at different circumstances and dimensions with intensity information making use of inherent variations in intensity and Relative Total Variation, seed point obtained from decomposition and co-occurrence features of statistical phenomena $[10,13]$ it is possible to detect the objects in camouflage images. The approach in this work is constructed and characterized with the help of multi resolution analysis, statistical characteristics; region growing by maximum intensity distance [4] given by D. Kroon and by discontinuity based segmentation invented to determine the sudden changes in image intensity over a minute value due to the image is having both BG and FG are almost similar in intensity[9]. Relative Total variation is applied in the approach in order to normalize the statistical data in image sequence due to sudden and once-off changes there after getting features of decomposed image using wavelet transform [10]. Using the set of statistical features the classification of foreground and background can be done thereby the camouflaged object is identified.

\section{METHODS TO IDENTIFY CAMOUFLAGED OBJECTS}

A) Identification Using Wavelet Coefficients and Statistical Features: There are different techniques to identify camouflaged objects but only few like: Convexity based and pattern based meet the requirements but there is no single point of solution so here contributing towards solution. In the proposed one selected fixed window approach has methods like multi resolution analysis, statistical characteristics based seed block selection and traditional region growing approach. In multi resolution analysis so many efficient and advanced decomposition techniques are available but in this analysis we are using the method proposed by Daubechies. Daubechies wavelets frequently used in solving different types of problems, e.g. self-similarity properties, and signal discontinuities, etc. The co-occurrence wavelet coefficients of sub blocks in resized image of $\mathrm{I}(p, q)[3]$ are highly correlated with each other and the similarity is due to the different features between the lines, edges and corners, textural patterns, shapes and size of objects. The joint probability gray level function in two dimension is known as the co-occurrence matrix $c(p, q),[13]$ it is used to illustrate the spatial similarity between the coefficients in wavelets to extract statistical characteristics. We know the GLCM and its features variants which were suggested by Harlick in 1973. From the intensity level correlated data measuring the features with following quantitative approaches.

$$
\begin{aligned}
& \text { NormalizedGLCM }=V(p, q)=\frac{c(p, q)}{\sum_{p, q=1}^{N-1} c(p, q)} \\
& \text { Contrast }=\sum_{p, q=1}^{N}(p-q)^{2} V(p, q) \\
& \text { Clustershade }=\sum_{p, q=1}^{N}\left(p-D_{x}+q-D_{y}\right)^{3} V(p, q) \\
& \text { Clusterpro min ence }=\sum_{p, q=1}^{N}\left(p-D_{x}+q-D_{y}\right)^{4} V(p, q) \\
& \text { Where } D_{x}=\sum_{p, q=1}^{N} p V(p, q) \\
& D_{y}=\sum_{p, q=1}^{N} q V(p, q)
\end{aligned}
$$


These feature values given in equation 1 are normalized in either with linear approach or logarithmic approach based on their outcomes ranges. An optimal edge detection approach is chosen to detect wide range of sudden variations in intensity instead of sobel and prewitt because of less number of sudden changes in image intensities also verified with canny edge methods which is one of the optimum solution, then applying region growing based on maximum Intensity distance measure. The functional flow of this approach is shown in figure3.1. The simulation results will depict how the objects are detected in coming sections.

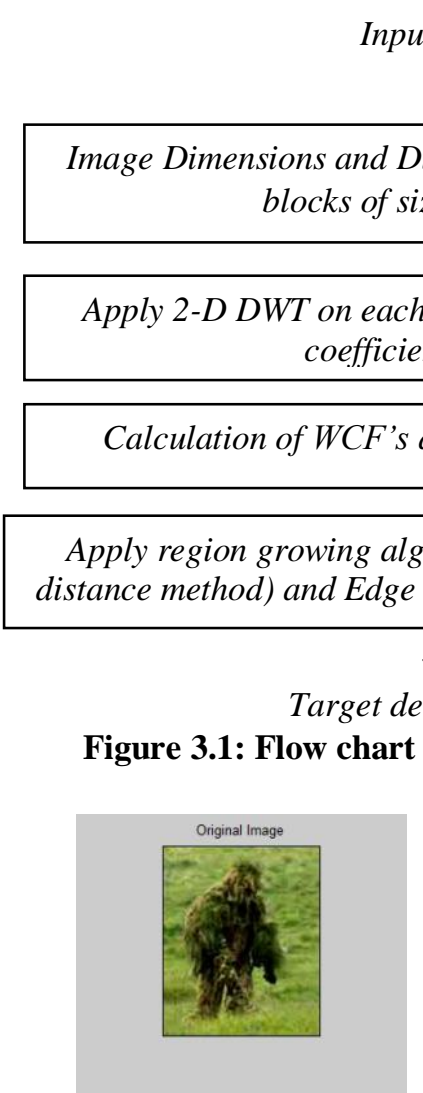

(a)

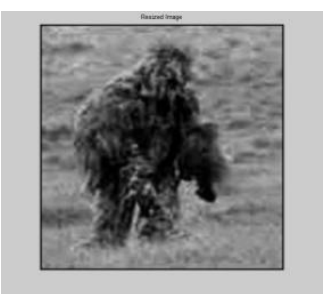

(b)
Figure 3.2 :(a) Original image of size 180x150x3,(b) Resized image of size 512 x512

The Constructive approach is initially image is resized with interpolation in order to suitable for algorithm for any kind of dimensions like $512 \times 512,256 \times 256$ and $312 \times 256$ e.t.c. After resizing the image is to be subdivided into non overlapping segments like:

Process of making sub-block Algorithm:

Step1: $A=0 ; p=1 ; q=32 ; r=1 ; s=32$;

Step2: for $i=1: 16$

Step3: if $(i>1)$ p,q, $r$ and $s$ updated by 32;

Step4:forj=1:16

$d(:,:, i, j)=b(p: q, r: s) ; A=A+1 ; s u b \_$image $(:,:, A)=d(:,:, i, j)$;

Each segment (non-overlapping sub block) is to be decomposed using two-dimensional wavelets for Multiresolution analysis. After decomposition each subblock features are added thereafter highest sum block is treated as seed block and center pel is seed point . The statistical features of these 256 sub blocks profile as represented in figure 3.3 illustrates the sporadic and non sporadic blocks in camouflage image there by with the help of seed point applying region growing using maximum intensity distance method and edge detection of object using traditional methods. Finally the object detection is found to be satisfactory but the drawback is fixed window technique and the method has a drawback when different characteristics entities (specially texture)
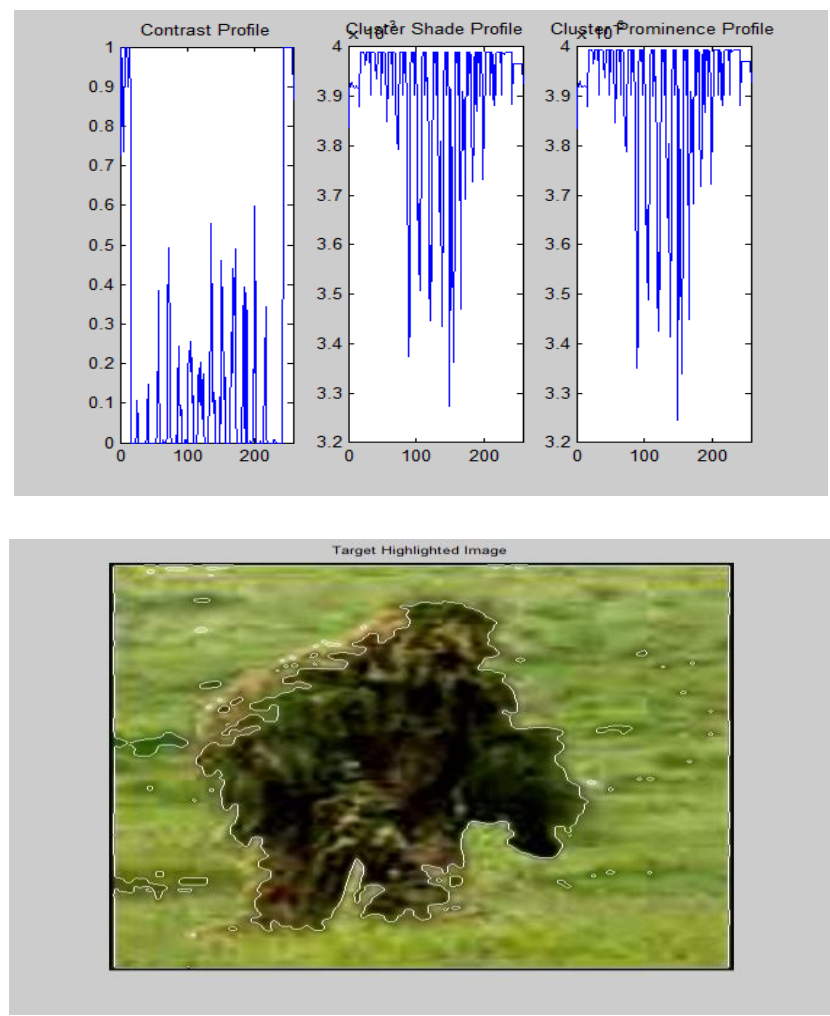

Figure 3.3: Profiles of statistical coefficient features and Target highlighted image

B) Identification using Texture measures: Statistical characterization based approach for the detection of object in camouflaged region for computer vision applications is not accurate because some clusters which are of not interested were recognized with the objects. The analysis of clusters identification was observed by testing on different kinds of camouflaged images. To mitigate these problems of fixed window length based statistical features approach considered the characteristics entity (especially colour, shape and texture e.t.c) smoothing (texture) in camouflaged image texture thereby applying the decomposed statistical features to detect the objects. The characteristic entity (texture) smoothing can be done like:

\section{Algorithm for Detection after smoothing characteristic entity:}

Initialization inputs (Ground truths): $\eta$ (Degree of Smooth) $0.03, \sigma$ (size of primitive element) [3x3], e (Edge Sharpness)[0.02], N (number of iterations)[6], $\varepsilon$ (Numerical stability), "S" is structure and "I" is input image.

Step 1: Finding pixel wise windowed total variation $\mathbf{W}$ with bivariate Gaussian window $(\mathrm{G})$

$$
W_{x}(p)=\sum_{\mathrm{q} \in \mathrm{R}(\mathrm{p})} \mathrm{G}_{\mathrm{p}, \mathrm{q}}\left|\left(\partial_{x} s\right)_{q}\right|
$$




$$
W_{y}(p)=\sum_{\mathrm{q} \in \mathrm{R}(\mathrm{p})} \mathrm{G}_{\mathrm{p}, \mathrm{q}}\left|\left(\partial_{y} s\right)_{q}\right|
$$

Step 2: Finding windowed inherent variation $\mathbf{W}_{\mathbf{I}}(\mathbf{p})$ by considering absolute value in step1 which can give overall spatial variation because pixel wise total variation can be positive or negative.

$$
W_{I_{x}}(p)=a b s\left(W_{x}(p)\right) \text { and } W_{I_{y}}(p)=a b s\left(W_{y}(p)\right)
$$

Step 3: Removing texture from an image by non linear weighting equation modelled by

$[\mathrm{W}, \mathrm{u}]=\mathrm{W} /\left(\mathrm{W}_{\mathrm{I}}+\varepsilon\right)$

Step 4: For stability of this nonlinear weighting equation the equation was decomposed in to linear equation parts by a iterative optimization procedure as $\left(1+\eta \mathrm{L}^{\mathrm{N}}\right) \mathrm{e}=\mathrm{I}$ Here $\mathrm{L}$ is weight matrix from $\mathrm{W}, \mathrm{u}$ "S" is structure "I" is input

$\mathbf{L}$ consist of $\mathrm{W}, \mathrm{u}$ and discrete gradient operators along horizontal and vertical with forward difference to approximate gradients

Step 5: The resultant texture normalized image is applied to statistical characterizer to detect object in camouflaged image

The algorithm mainly involved with sparse diagonalization and conditioned conjugate gradient methods to solve linear models form weights of characteristic entity. By applying the characteristic entity smoothing with statistical characterization, the detection of object in camouflaged image analyzed under different environments like:

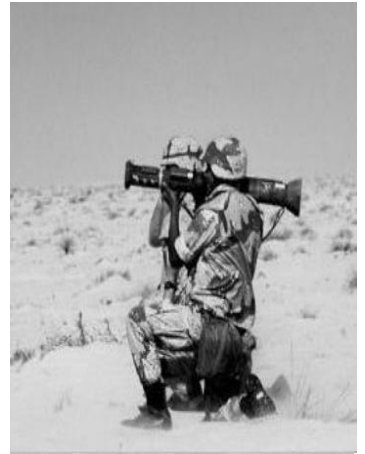

(a)

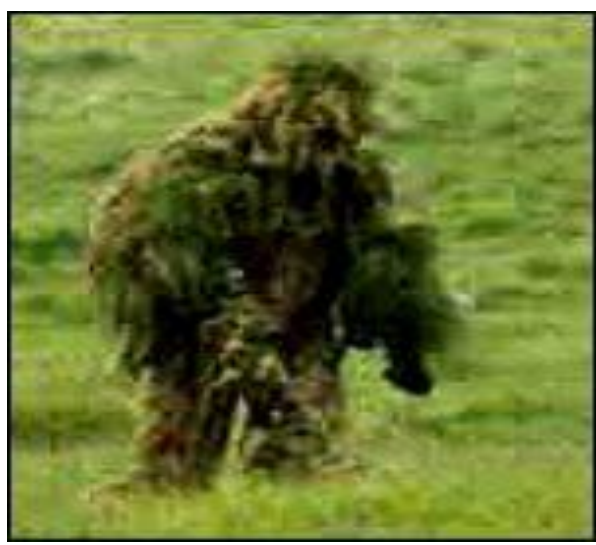

(c)

Figure 3.4 Input images (a) Camouflage Soldier (b) Camouflage Aero Plane (c) Camouflage Soldier in Grass Fields

\section{RESULTS AND DISCUSSIONS}

The above discussed methods for object detection under camouflaged images and corresponding algorithms were simulated in Matlab (7.7). The input images may be colour or gray scale is of any resolution. These images contain different categories of targets such as insects, soldiers in desert war environment, soldier in grass fields, camouflaged tankers, aero planes, birds and statues which are collected to verify the detection performance of the method (A) and method (B)

The analysis of object detection using $\operatorname{Method}(A)$

Target Extracted Image

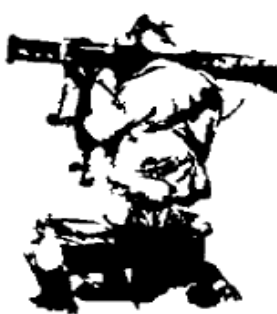

(a)

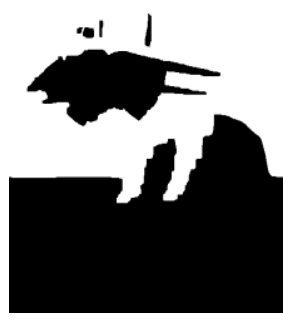

(b)

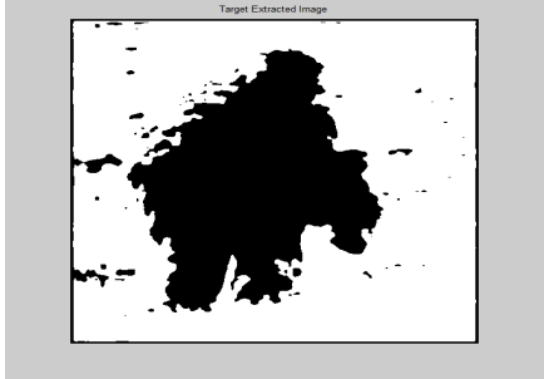

(c)

The analysis of object detection using Method (B)

Figure (4):(a) camouflage soldier in desert environment detection using statistical features from method $A$ and detection by smoothing texture measures from method B. (b) camouflage Aero Plane detection using statistical features from method $A$ and detection by smoothing texture measures from method B. (c) camouflage soldier in grass fields detection using statistical features from method $A$ and detection by smoothing texture measures from method $B$.

The performance analysis of these two methods to detect camouflaged objects assessed by regular metrics for image segmentation like precession and recall from true positives, true negatives, false positives and false negatives $[6,7,8,9]$ in target extracted outputs shown in figure(4). The test images corresponding statistical features using method $\mathrm{A}$ and method $\mathrm{B}$ are listed in tabular representation in table(1)

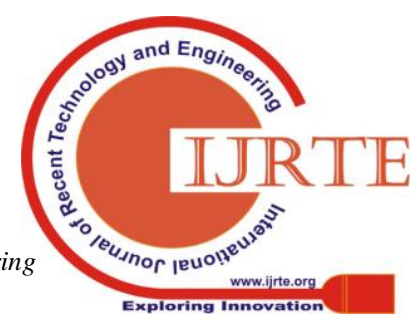




\begin{tabular}{|l|c|c|c|c|c|}
\hline \multicolumn{1}{|c|}{ Image } & $\begin{array}{c}\text { Seed } \\
\text { Block }\end{array}$ & $\begin{array}{c}\text { Seed } \\
\text { Point }\end{array}$ & $\begin{array}{c}\text { Co } \\
\text { ntr } \\
\text { ast }\end{array}$ & $\begin{array}{c}\text { Cluster } \\
\text { shade }\end{array}$ & $\begin{array}{c}\text { Cluster } \\
\text { promin- } \\
\text { ence }\end{array}$ \\
\hline $\begin{array}{l}\text { Camoufl- } \\
\text { aged } \\
\text { soldiers } \\
\text { in desert }\end{array}$ & 107 & $\begin{array}{c}(209, \\
337)\end{array}$ & 750 & $\begin{array}{c}1.287 \mathrm{e}+0 \\
11\end{array}$ & $\begin{array}{c}1.436 \mathrm{e}+0 \\
14\end{array}$ \\
\hline $\begin{array}{l}\text { Camoufl- } \\
\text { aged } \\
\text { soldier in } \\
\text { fields }\end{array}$ & 122 & $\begin{array}{c}(299, \\
38)\end{array}$ & 387 & $\begin{array}{c}1.279 \mathrm{e}+0 \\
11\end{array}$ & $\begin{array}{c}3.039 \mathrm{e}+0 \\
14\end{array}$ \\
\hline
\end{tabular}

The constructive approach on different environments shows the result

\begin{tabular}{|l|c|c|c|c|c|}
\hline $\begin{array}{l}\text { Aero } \\
\text { plane }\end{array}$ & 113 & $(113,115)$ & 87 & $\begin{array}{c}2.9077 \mathrm{e} \\
+011\end{array}$ & $\begin{array}{c}1.4506 \\
\mathrm{e}+014\end{array}$ \\
\hline Bird & 113 & $(119,205)$ & 302 & $\begin{array}{c}1.480 \mathrm{e}+ \\
011\end{array}$ & $\begin{array}{c}3.6802 \\
\mathrm{e}+011\end{array}$ \\
\hline $\begin{array}{l}\text { Statu } \\
\text { e }\end{array}$ & 203 & $(237,205)$ & 209 & $\begin{array}{c}1.4434 \mathrm{e} \\
+011\end{array}$ & $\begin{array}{c}2.0414 \\
\mathrm{e}+014\end{array}$ \\
\hline Ship & 117 & $(233,373)$ & 335 & $\begin{array}{c}3.336 \mathrm{e}+ \\
010\end{array}$ & $\begin{array}{c}8.6129 \\
\mathrm{e}+012\end{array}$ \\
\hline
\end{tabular}

Table 1: Statistical features of different kinds of camouflage images

The image segmentation of object detection approach performance can be analysed in many ways like standard measures, distance measures and similarity measures. Standard measures are the best suitable ones in comparison with similarity and distance in case of camouflaged approach because foreground and background pixels are of same characteristic entities(like: intensity colour and texture e.t.c). To access the performance here considered three indices from standard measures are Precision Recall and F-Measure [19] which are depending on confusion matrix entries.

Precision $(\mathrm{P})=$ True positives/(True Positive+ False positives)

Recall $(\mathrm{R})=$ True positives/ (True Positive+ False Negatives)

and $\mathrm{F}$-measure $=2 * \mathrm{P} * \mathrm{R} /(\mathrm{P}+\mathrm{R})$

The problem of camouflage is sporadic in image or sequence of image here we considered specific frames where the rate of camouflage is more while detecting objects using statistical features set (Method A) and constructive one of both statistical features in smoothed characteristic entities(Method B).

\begin{tabular}{|l|c|c|c|}
\hline $\begin{array}{c}\text { Input and } \\
\text { Performance } \\
\text { (Standard } \\
\text { measure) }\end{array}$ & $\begin{array}{c}\text { Input image } \\
\text { (a) } \\
\text { Camouflage } \\
\text { Soldier in } \\
\text { war } \\
\text { environments }\end{array}$ & $\begin{array}{c}\text { Input image } \\
\text { (b) }\end{array}$ & $\begin{array}{c}\text { Input } \\
\text { Camouflage } \\
\text { Amage }(\mathrm{c}) \\
\text { Camouflage } \\
\text { Soldier in } \\
\text { Grass } \\
\text { Fields }\end{array}$ \\
\hline Precision & 0.902 & 0.878 & 0.976 \\
\hline Recall & 0.924 & 0.761 & 0.573 \\
\hline F Measure & 0.90 & 0.815 & 0.74 \\
\hline
\end{tabular}

Table 2: Performance evaluation on three kinds of images

\section{Performance analysis:}

The performance analysis of method A and method B defines that it is necessary that smoothing of characteristic entity and statistics of smoothed entity gives better detection than that of method A. Both of the methods gives satisfactory results on several kinds of images but if the intermittent is more in camouflaged image, then method B is better compared with method A.

\section{CONCLUSIONS AND FUTURE SCOPE}

In the proposed work mainly developed a constructive approach to mitigate the problems in conditions like camouflage and the proposed one tested on different kinds of images to validate the method. Multiresolution based approach is chosen because it has wide range of properties and gives potential solution in target detection applications. Wavelet coefficient feature of sub blocks are used as profile to identify the seed point in this implementation. Also implemented a characteristic entity (texture) smoothed and their statistical features to detect object in camouflaged images more effectively. Performance analysis of the algorithms verified on images of arbitrary dimensions, intensities variations on images, and pertaining to various conditions and the solutions are acceptable. In future the resizing and decomposition levels are depend on size of the target and also dimension of image with many informative objects mingled in image are considerable research work to carry a object detection methods in an optimum detection. Depends on feature sets and multiple target detection is also a challenging problem in camouflage image.

\section{REFERENCES}

1. Sujit K Singh, Chitra A Dhawale and Sanjay Misra, "Survey of Object Detection Methods in Camouflaged Image", Proc: IERI, PP. 1-6, ELSEVIER, 2012

2. Yuxin Pan, Yiwag Chen, Qiang Fu, Ping Zhang \& Xin Xu, "Study on the Camouflaged target Detection Method Based on 3D Convexity", Proc: Modern Applied Science, Vol. 5, PP. 152-157, 2011.

3. Dileep Kumar, Dr. Shrishu Varma, "Multiresolution framework with Neural Network approach for Automatic Target Recognition (ATR)", Proc: ICSAAP, PP. 168-173, IEEE computer society, 2009.

4. Li Xu Qiong Yan Yang Xia Jiaya Jia "Structure Extraction from Texture via Relative Total Variation" ACM Transactions on Graphics, Vol. 31, No. 6, Article 139, November 2012.

5. S. Z. Mahmoodabadi, A. Ahmadian, M. D. Abolhasani, M. Eslami, J. H. Bidgoli, "Feature Extraction Based on Multiresolution Wavelet Transform", IEEE proceedings, PP. 3902-3905, 2005.

6. Zeyong Shan and Selin Aviyente, "Image Denoising Based on the Wavelet Co-Occurrence matrix", Proc:ICASSP2005 , PP. 645-648, IEEE 2005

7. Hung-Kuo Chu, Wei-Hsin Hsu, Niloy J. Mitra, Daniel Cohen-Or, Tien-Tsin Wong and Tong-Yee Lee "Camouflage Images" ACM Transactions on Graphics.

8. Yaser Sheikh and Mubarak Shah "Bayesian Modeling of Dynamic Scenes for Object Detection" IEEE transactions on pattern analysis and machine intelligence, vol. 27, no. 11, november 2005

9. G. Van de Wouwer, P. Scheuders and D. Van Dyck, "Statistical Texture Characterization from Discrete Wavelet Representations" Proc: IEEE transactions on image processing, vol.8, pp. 592-598, 1999.

10. Zeyong Shan and Selin Aviyente, "Image Denoising Based on the Wavelet Co-Occurrence matrix", Proc:ICASSP2005 
, PP. 645-648, IEEE 2005.

11. Robert M. Haralick, K. Shanmugam and Itshak Dinstein, "Textual Features for Image Classification", Proc: IEEE transactions on systems, man, and cybernetics, vol smc-3, no.6, PP. 610-621, 2010.

12. G. Van de Wouwer, P. Scheuders and D. Van Dyck, "Statistical Texture Characterization from Discrete Wavelet Representations" Proc: IEEE Transactions On Image Processing , Vol.8, PP. 592-598, 1999

13. Ch. S. Sastry, Arun K. Pujari, B. L. Deekshatulu, C. Bhagvati, "A Wavelet based Multiresolution algorithm for rotation invariant feature extraction", Proc: Pattern Recognition Letters, vol. 25, PP. 1845-1855, ELSEVIER Pattern Recognition Letters 2004.

14. Sirui Tian, Chao Wang, Hong Zhang, Bo Zhang, Fan Wu, "A Wavelet Based Targets Detection Method for high resolution Airborne SAR Data ", Proc: IEEE transactions of Geosciences and Remote Sensing Symposium , PP. 2071-2073, 2007.

15. Bir Bhanu, Automatic Target Recognition: State of the Art Survey, IEEE Transactions on Aerospace and Electronic Systems, Vol. AES-22, No. 4, July 1986

16. Rafael C. Gonzalez and Richard E. Woods, "Digital image processing", Pearson education, Inc, 1999.

17. E. Hosseini, Aria M.R. Saradjian, J. Amini and C. Lucas, "Generalized co-occurrence Matrix to Classify IRS-1D Images using Neural Network", 20th ISPRS Congress, Istanbul, Turkey, July 2004

18. Michael W. Roth, "Survey of Neural Network Technology fot Automatic Target Recognition", IEEE Transactions on Neural Networks.

19. Xiang Zhang, Ce Zhu, Shuai Wang, Yipeng Liu and Mao Ye “ A Bayesian approach for camouflaged moving object detection"IEEE Transactions on Circuits and Systems for Video Technology Year: 2016, Volume: 09

20. Liyuan Li, Weimin Huang, Irene Yu-Hua Gu, and Qi Tian "Statistical Modeling of Complex Backgrounds for Foreground Object Detection" IEEE Transactions On Image Processing, Vol. 13, No. 11, November 2004.

\section{AUTHORS PROFILE}

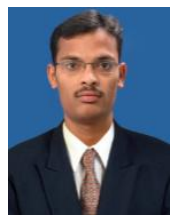

Chennamsetty Pulla Rao received his Bachelors of Degree in Electronics and Telecommunication Engineering from IETE in 2007. Received Master's degree in Advanced Communication Systems from National Institute of Technology, Warangal in 2010. At present working as Associate Professor in MIC College of technology from July 2010.Published one National and one International Journal research interests are image processing techniques and Wireless Communications.

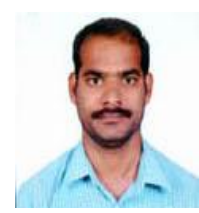

Dr. A Guruva Reddy working as Principal and Professor in Electronics \& communication engineering at Universal College of Engineering in INDIA. Completed Doctorial degree from JNTU Ananthapur in the year of 2010. He has 15 international and national journals in the area of image processing. Also organized and attended national level workshops like Funded by SERB and Faculty Development programmes. At present supervising five research scholars in the area of signal and image processing at JNTU Kakinada.

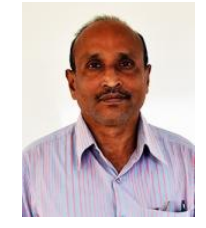

Dr. Rama Rao C B working as Professor in Electronics \& communication engineering at NIT Warangal in INDIA. Received Doctorial degree from IIT kharagpur in the year of 1995. Published 12 international and national journals in the area of signal and image processing. Also organized and attended several national level workshops like ISTE and STTP. At present supervising three research scholars in the area of signal and image processing at NIT Warangal. 